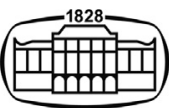

AKADÉMIAI KIADÓ

\title{
A potential role for psilocybin in the treatment of obsessive-compulsive disorder
}

EDWARD JACOBS ${ }^{1,2 *}$ (1)

\section{Journal of Psychedelic Studies}

4 (2020) 2, 77-87

DOI:

$10.1556 / 2054.2020 .00128$

(c) 2020 The Author(s)

\section{ORIGINAL ARTICLE}

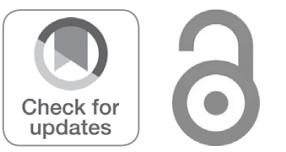

${ }^{1}$ Centre for Affective Disorders, Institute of Psychiatry, Psychology and Neuroscience, Kings College London, London, UK

${ }^{2}$ Department of Psychiatry, University of Oxford, Oxford, UK

Received: January 03, 2020 - Accepted: June 16, 2020

Published online: July 17, 2020

\begin{abstract}
The recent revivification of interest in the therapeutic use of psychedelics has had a particular focus on mood disorders and addiction, although there is reason to think these drugs may be effective more widely. After outlining pertinent aspects of psilocybin and obsessive-compulsive disorder (OCD), the current review summarizes the evidence indicating that there may be a role for psilocybin in the treatment of OCD, as well as highlighting a range of potential therapeutic mechanisms that reflect the action of psilocybin on brain function. Although the current evidence is limited, that multiple signals point in directions consistent with treatment potential, alongside the psychological and physiological safety of clinically administered psilocybin, support the expansion of research, both in animal models and in further randomized controlled trials, to properly investigate this potential.
\end{abstract}

\section{KEYWORDS}

obsessive compulsive, OCD, psilocybin, psilocin, psychedelic

\section{INTRODUCTION}

The last decade has witnessed a resurgence of interest in serotonergic psychedelics, such as lysergic acid diethylamide (LSD) and psilocybin, for the treatment of a range of psychopathologies. Whilst individuals self-medicate with (typically illegally procured) drugs, universities are undertaking controlled clinical trials with legal authorization from governments. Although the best-developed of these research programmes have focused on the treatment of mood disorders and addiction, the aim of this review is to discuss the potential use of psilocybin for patients with Obsessive-Compulsive Disorder (OCD). Tentative evidence from multiple sources (preclinical investigations, case studies, and an open-label trial) together suggest that psilocybin may be an effective treatment and, given the compound's safety when administered in a medically-controlled context, further investigation is warranted.

\section{PSILOCYBIN}

\section{Background}

*Corresponding author. Centre for Affective Disorders, Institute of Psychiatry, Psychology and Neuroscience, Kings College London, London, UK

E-mail: eddie.jacobs@kcl.ac.uk
Psilocybin (IUPAC name [3-(2-dimethylaminoethyl)-1H-indol-4-yl] dihydrogen phosphate) is a naturally occurring psychoactive compound found in the sclerotia and fruiting bodies of approximately 200 mushroom species worldwide. Humans have been ingesting psilocybincontaining mushrooms for hundreds, if not thousands of years, interpreting the resulting perceptual anomalies and altered sense of reality as a means for communing with the spirits of the natural world in order to obtain healing and knowledge (Hofmann \& Schultes, 1979). Nonetheless, since 1971 psilocybin has been in Schedule I of the United Nations Single Convention on Psychotropic Substances, and as such, both recreational and scientific use is 
subject to the strictest control in jurisdictions worldwide, including Schedule I of the United States' Controlled Substances Act, as well as Class A and Schedule 1 of the United Kingdom's Misuse of Drugs Act.

\section{Pharmacology \& neurobiology of psilocybin}

A tryptamine alkaloid, psilocybin was first isolated from the Psilocybe mexicana in 1957, and synthesized the following year, by the discoverer of LSD, chemist Albert Hofmann (Hofmann, Frey, Ott, Petrzilka, \& Troxler, 1958). When taken orally, it is rapidly dephosphorylated in the acidic environment of the stomach, and by alkaline phosphatases in the intestine, to produce psilocin, a lipid-soluble phenol compound which easily crosses the blood-brain barrier (Hasler, Bourquin, Brenneisen, Bär, \& Vollenweider., 1997). Early preclinical studies demonstrated that the behavioral effects of psilocybin parallel the increase in the level of psilocin in the brain (Horita, 1963). Although psilocin is a full or partial agonist at a range of receptors (Halberstadt \& Geyer, 2011; Ray, 2010), it shows notable affinity for the serotonin $5-\mathrm{HT}_{1 \mathrm{~A}}, 5-\mathrm{HT}_{2 \mathrm{~A}}$, and $5-\mathrm{HT}_{2 \mathrm{C}}$ sub-types.

Although the $5-\mathrm{HT}_{1 \mathrm{~A}}$ and $5-\mathrm{HT}_{2 \mathrm{C}}$ receptors are known to play some role in the effects of psilocin (Halberstadt, Koedood, Powell, \& Geyer, 2011), the 5- $\mathrm{HT}_{2 \mathrm{~A}}$ receptor principally mediates its characteristic effects. In humans, subjective intensity following psilocybin administration correlates with $5-\mathrm{HT}_{2 \mathrm{~A}}$ receptor occupancy (Madsen et al., 2019), while pre-treatment with a selective $5-\mathrm{HT}_{2 \mathrm{~A}}$ antagonist blocks, or significantly attenuates, the characteristic effects of psilocybin (Kometer et al., 2012; Quednow, Kometer, Geyer, \& Vollenweider, 2012; Vollenweider, VollenweiderScherpenhuyzen, Bäbler, Vogel, \& Hell, 1998).

While full accounts of the downstream neural effects of these changes are available elsewhere (Carhart-Harris, 2019; Nichols, 2016; Vollenweider \& Kometer, 2010), the most salient consequences of $5-\mathrm{HT}_{2 \mathrm{~A}}$ activation by a psychedelic include enhancement of activity of neuronal networks in the prefrontal cortex via an elevated but asynchronous release of glutamate (Aghajanian \& Marek, 1999; Béïque, Imad, Mladenovic, Gingrich, \& Andrade, 2007; Muschamp, Regina, Hull, Winter, \& Rabin, 2004).

Enhanced glutamatergic activity may lead to increased expression of brain-derived neurotrophic factor (BDNF) (Baumeister, Barnes, Giaroli, \& Tracy, 2014; Vollenweider \& Kometer, 2010), thought to play a role in adult neurogenesis (Waterhouse et al., 2012), and psychedelics increase neuritogenesis and spinogenesis both in vitro and in vivo (Ly et al., 2018). The asynchronous nature of psychedelic-induced glutamate-release at the single-neuron level leads to disruptions to the normal rhythmic oscillations of cortical neurons, and ultimately to the disorganisation of cortical activity (Carhart-Harris et al., 2012; Muthukumaraswamy et al., 2013), and temporary disintegration of resting-state networks such as the Default Mode Network (DMN) (Carhart-Harris et al., 2016; Muller, Dolder, Schmidt, Liechti, \& Borgwardt, 2018; Muthukumaraswamy et al., 2013; Palhano-Fontes et al., 2015). This disintegration within networks is coupled with desegregation between networks (i.e., increased global connectivity - Tagliazucchi et al., 2016). These network-level phenomena are associated with subjective reports of "ego dissolution" and the "peak" or "mystical" experience, wherein users report a breaking down of the distinction between subject and object, alongside a sense of sacredness, of "transcending time and space", and a deeply felt positive mood (Pahnke, 1966, 1969). Larger doses of psilocybin are associated with greater likelihood of peak experience (Griffiths et al., 2011), and multiple studies have reported that peak experiences are predictive of therapeutic effect (reviewed in Majić, Schmidt, \& Gallinat, 2015), as discussed below.

\section{Characteristic use and effects of psilocybin}

After oral administration of $10-20 \mathrm{mg}$ of psilocybin, peak plasma levels of psilocin are reached after approximately 105 minutes. Physiological and subjective effects begin within 20-90 minutes (Hasler, Grimberg, Benz, Huber, \& Vollenweider, 2004), and persist up to 6 hours. Typical effects include dramatic changes to cognition and perception including illusions and synesthesia, as well as distortions to perception of self, time, and space, often accompanied by heightened affect. Adverse events recorded in recent clinical trials include transient hypertension, nausea and vomiting, headache, and confusion (Studerus, Kometer, Hasler, \& Vollenweider, 2011). Serotonergic psychedelics may exacerbate pre-existing psychosis, and for this reason, modern studies have excluded those with a history, or family history, of a psychotic condition (Johnson, Richards, \& Griffiths, 2008). Extreme psychological distress, and risky behavior, are certainly possible with the use of psychedelics, although these risks can be minimized and managed through (i) judicious attention to "set" and "setting" - the psychological and environmental context for a psilocybin experience (Carhart-Harris et al., 2018a; Hartogsohn, 2016; Leary, Litwin, \& Metzner, 1963), and (ii) the use of a sober "minder" or "guide" - a harm-reduction strategy often used by recreational users, and always employed in clinical contexts (Johnson, Richards, \& Griffiths, 2008).

Drug effects increase monotonically with dose, with doses as low as $5 \mathrm{mg} / 70 \mathrm{~kg}$ producing subjectively perceptible effects (Griffiths et al., 2011). Active doses in recent experimental and clinical studies have been in the range of 10-35 mg per os. Typical recreational doses vary from 10 to $50 \mathrm{mg}$, approximately equivalent to $10-50 \mathrm{~g}$ of fresh mushrooms, or 1-5 g of dried mushrooms. However, the natural variability of psilocybin content, and the content of other psychoactive alkaloids, across different species and even different batches of Psilocybe mushrooms, mean that users risk some inaccuracies in their dosing. Although there remain very real risks of users coming to harm during "bad trips" in uncontrolled settings (Carbonaro et al., 2016), the drug is associated with particularly low rates of emergency treatment seeking (Winstock, Barratt, Maier, \& Ferris, 2018).

In public consciousness, magic mushrooms have a negative image associated with mental ill-health, in part because of a now-recognized risk of exacerbating psychotic conditions, 
but also due to a sensationalized cultural history (Lee \& Shlain, 1985). However, two recent large scale retrospective population studies, including separate data sets of over 130,000 US adults, not only failed to find any evidence of an association between psychedelic use and mental health problems, but actually found lower likelihood of impaired mental health and suicidality following psychedelic use (Johansen \& Krebs, 2015; Krebs \& Johansen, 2013), a finding replicated for psilocybin specifically in an independent analysis (Hendricks, Thorne, Clark, Coombs, \& Johnson, 2015). Given the rigorous screening and preparation process in experimental and clinical trials, in which great efforts are made to familiarize and reassure participants about likely drug effects, along with the high level of clinical care and attention afforded during drug sessions, recent studies have been associated with very low incidences of extreme psychological distress: although sedative medications have been included in the standard safety protocols of recent studies, there is as yet no recorded mention of their use.

\section{Clinical efficacy}

Although some 40,000 patients were treated with psychedelics in the 1950s and 1960s, with often positive results recorded in more than 1,000 clinical papers (Grinspoon \& Bakalar, 1979), the greater part of this research - like most research from that period - does not stand up to modern standards of descriptive, methodological, or analytical rigor (Rucker, Jelen, Flynn, Frowde, \& Young, 2016). Nonetheless, recent investigations of psilocybin have provided tentative but growing evidence for its promise in the treatment of mood disorders, addiction, and OCD.

Currently, the best-explored indication for psilocybin has been in the treatment of anxiety and depression secondary to cancer, or terminal diagnoses. While mortality-related existential despair is not a recognized condition in DSM-V, depression and anxiety secondary to cancer and other terminal illnesses appear to respond comparatively poorly to standard pharmacotherapies (Grassi, Caruso, Hammelef, Nanni, \& Riba, 2014; Ostuzzi, Benda, Costa, \& Barbui 2015). A small trial of patients with advanced-stage cancer with a comorbid anxiety disorder (Grob et al., 2011) found that a single modest dose $(14 \mathrm{mg} / 70 \mathrm{~kg})$ of psilocybin was sufficient to reduce trait-anxiety at 1 and 3 months after treatment, as well as a significant reduction in depressive symptoms, as measured by the Beck Depression Inventory (BDI), at 6 months. Later studies (Griffiths et al., 2016; Ross et al., 2016) employed double-blind, cross-over trials of a higher dose (21-22 mg/70 kg) of psilocybin in patient groups of 29 and 51 cancer patients with a DSM-IV depression or anxiety diagnosis, tested against active placebos of a very low dose of psilocybin (1-3 mg/70 kg) or niacin $(250 \mathrm{mg})$. As with the vast majority of recent clinical research using psychedelics, both protocols conceived of the treatment as a combination pharmaco-psychotherapy approach. On drug dosing days, patients wore eyeshades and listened to music, with lighttouch, supportive, and largely non-directive contributions from therapists. Dosing days were embedded within wider psychotherapy programmes, with preparatory sessions to establish therapeutic rapport and reassurance about drug effects, as well as integrative sessions to make sense of experiences under drug effects. Ross et al. found that psilocybin produced rapid and enduring decreases in anxiety and depression that persisted for $60-80 \%$ of patients at the 6.5 month mark, as well as significant improvements in demoralization, hopelessness, and attitudes towards death. These effects were replicated by Griffiths et al., with patients displaying clinically significant reductions in clinician-measured and self-rated measures of anxiety and depressed mood at the 6-month follow-up. These were accompanied by significant persisting effects on positive attitudes about life and self, as well as improved relationships. Notably from both these studies, improvements at 5 or 6 weeks in a suite of measures of depression, anxiety, and outlook, significantly correlated to the extent of a patient's 'mystical experience' during the psilocybin session, as measured by the Mystical Experience Questionnaire (MEQ30) (Barrett, Johnson, \& Griffiths, 2015).

The apparent therapeutic importance of the quality of the psilocybin experience has been suggested elsewhere. In an open-label trial of psilocybin for 19 patients with treatmentresistant depression, patients underwent two drug sessions in a supportive setting, of $10 \mathrm{mg}$ and later $25 \mathrm{mg}$ psilocybin, separated by a week (Carhart-Harris et al., 2018b). All patients showed some reduction in depression severity 1 week after the high-dose session, with clinically significant reductions sustained by most patients for 3-5 weeks, and in many for up to 6 months. Notably, improvements in depression severity at 5 weeks were predicted by the extent to which the $25 \mathrm{mg}$ session induced an experience of "oceanic boundlessness," a dissolution of ego boundaries associated with positive mood (Roseman, Nutt, \& Carhart-Harris, 2018).

Similar patterns of promising results have been recorded in open-label studies of psilocybin in substance use disorders. Two sessions with psilocybin $(21-28 \mathrm{mg} / 70 \mathrm{~kg}$ ) within a wider context of Motivational Enhancement Therapy led to significant increases in alcohol abstinence in problem drinkers, with results largely persisting to 36-week follow-up. MEQ scores (but also general drug intensity) in the first session were predictive of improvements in number of drinking days, alcohol craving, and abstinence self-efficacy (Bogenschutz et al., 2015). Elsewhere, a sample of 15 nicotine-dependent smokers undertook two or three doses of psilocybin $(20-30 \mathrm{mg} / 70 \mathrm{~kg})$ embedded within a CBT-based smoking cessation therapy course. Biologically-verified smoking abstinence at 12-months was significantly higher than that produced by other available psychotherapies and pharmacotherapies (Johnson, Garcia-Romeu, \& Griffiths 2017), with successful abstainers scoring higher on a measure of mystical experience (Garcia-Romeu, Griffiths, \& Johnson, 2014).

\section{OCD}

\section{Burden of OCD}

OCD, a complex condition primarily characterized by anxiety-laden intrusive thoughts, images, or urges (obsessions), 
and repetitive behaviors which only temporarily reduce that anxiety (compulsions), is associated with significantly compromised quality of life (Coluccia et al., 2016). While estimates for the lifetime prevalence of OCD have ranged between 2 and 2.5\% (Fontenelle, Mendlowicz, \& Versiani, 2006; Ruscio, Stein, Chiu, \& Kessler, 2010), both behavioral and pharmacotherapies are recorded to have high nonresponse rates (Fisher \& Wells, 2005; Pittenger \& Bloch, 2014). Given that ablative lesion neurosurgery is used to treat intractable cases, despite not being recommended due to its inherent major risks, there is a real need to develop novel treatments.

\section{Pathophysiology of OCD}

Driven by findings from treatment studies as well as animal and genetic research, neurochemical models of OCD have focused on three candidate neurotransmitter systems - serotonin, dopamine, and glutamate (Graat, Figee, \& Denys, 2017). However, inconsistent responses to single monoamine-targeted treatments, as well as inconclusive evidence from pharmacologic challenge studies, weaken the case for straightforward pathophysiological models that cast OCD as primarily a disorder of monoamine function. While SSRIs are the typical first-line anti-obsessional agents, some $20-40 \%$ of OCD patients do not respond adequately to them (Denys, 2006), with reported symptom decreases of between 30 and $50 \%$ in those that do respond (Denys, van der Wee, van Megen, \& Westenberg, 2003). Additionally, dietary depletion of tryptophan - necessary for the biosynthesis of serotonin - does not lead to a worsening of symptoms in OCD (Berney, Sookman, Leyton, Young, \& Benkelfat, 2006). Other studies have shown positive treatment responses from dopamine antagonists in those refractory to treatment with SSRIs (McDougle, Epperson, Peloton, Wasylink, \& Price, 2000), with a number of studies pointing to a role for increased dopaminergic transmission in the maintenance of symptoms (reviewed in Westenberg, Fineberg, \& Denys, 2007). While these findings perhaps indicate a biological heterogeneity of OCD phenotype, it may be that dopaminergic and serotonergic modulations compensate, through different mechanisms, to some abnormality in a functionally coupled system underlying the neurobiology of OCD.

There is extensive interaction between the dopamine and serotonin systems, particularly in the basal ganglia (Parent, Wallman, Gagnon, \& Parent, 2011), an area consistently implicated in OCD by neuroimaging studies (Del Casale et al., 2011; Sakai et al., 2011; Welter et al., 2011). Functional changes to basal ganglia activity correlate with clinical improvement in OCD patients (Nakao, Okada, \& Kanbda, 2014), and insults to this area are known to produce OCD symptoms (Asbahr et al., 2005; Carmin, Wiegartz, Yunus, \& Gillock, 2002; Thobois, Jouanneau, Bouvard, \& Sindou, 2004).

In addition to abnormal basal ganglia activity, functional neuroimaging of OCD patients typically reveals increased activation in the orbitofrontal cortex (OFC) and anterior cingulate cortex (ACC), which are connected to the basal ganglia via cortico-striatal-thalamo-cortical (CSTC) circuitry, leading to a popular, though not definitive (see Menzies et al., 2008; Milad \& Rauch, 2012) account of the neural underpinnings of OCD. In the CSTC model, the OFC and ACC, the basal ganglia, and thalamus are connected in a circuit containing a direct loop via the globus pallidus pars interna, with a net excitatory effect on the thalamus, and an indirect loop via the globus pallidus pars externa and subthalamic nuclei, with a net inhibitory effect. It is proposed that, in OCD, an excess of activity through the direct pathway over the indirect pathway leads to a positive feedback loop between the OFC and ACC and the thalamus, resulting in disruptions to the thalamus' function in sensory gating. As a consequence, information that would otherwise be processed implicitly is subject to persistent conscious attention.

Alongside maladaptively persistent conscious attention to stimuli, OCD patients experience aversive emotional responses to them, including fear, disgust, and anxiety. Although there is not yet a widely accepted model of the neural circuitry underlying these affective responses, a recent meta-analysis of 25 neuroimaging studies (Thorsen et al., 2018) lends credence to the previously contested suggestion that they are subserved by hyperactivity in the amygdala, a brain area centrally implicated in the perception and generation of emotion.

Repeated conscious concerns about threat or contamination, coupled with exaggerated affective responses, lead to behaviors aimed at responding to the perceived threat. The transient relief that follows rewards and reinforces the behavior, leading to the cyclical recurrence of an obsession and its consequent compulsion.

\section{PSILOCYBIN AND OCD}

\section{Research supporting psilocybin's use in treating OCD}

Despite a paucity of clinical trials investigating the antiobsessional effect of psilocybin, converging evidence from a number of sources, as well as some possible mechanisms of action, lend support to its plausibility as a treatment. Although a considerable number of user reports on the internet - and one critically acclaimed stand-up comedy show, developed and performed by an OCD patient - attest to the efficacy of psilocybin, their low evidentiary weight preclude their exploration here. The varied evidence outlined below, although far from conclusive, indicate a triangulation of signals in support of a potential role for psilocybin in treating $\mathrm{OCD}$, meriting the extension of formal research investigating this possibility.

In a rodent model of OCD - marble-burying behavior Matsushima and colleagues (2009) studied the effects both of psilocybin, and a solution of powdered Psilocybe argentipes, in mice. While both synthetic psilocybin and the mushroom solution were recorded to reduce marble burying without reducing general locomotor activity, the study is beset with interpretive challenges. Starkly, there remain 
substantial grounds that a true animal model can be found of a condition primarily characterized by obsessive thoughts, which, if they exist in mice, cannot be accessed (Pittenger, Dulawa, \& Thompson, 2017). Although marble-burying tests have been widely used as a cost-effective model of OCD, on the grounds that a non-functional repetitive behavior may reflect the behavioral, outwardly measurable expression of OCD, the test shows poor predictive validity, notably because it is also responsive to anxiolytic drugs with no anti-compulsive activity (Taylor, Lerch, \& Chourbaji, 2017). But since psilocybin is well-established to have an anxiolytic effect (Griffiths et al., 2016; Ross et al., 2016), the causal process driving the positive effect reported by Matsushima et al. remains underdetermined. Elaborating on this finding by exploring anti-compulsive effect across multiple animal models would be of particular value.

Several case studies have detailed patients with OCD and similar diagnoses who have alleviated their symptoms with selfadministration of serotonergic psychedelics including Psilocybe mushrooms. Regrettably, none of these reports include labbased analyses of the drugs in question, so precise doses and amounts of active ingredients are unavailable. Both Leonard and Rapoport (1987) and Moreno and Delgado (1997) report male polydrug users with OCD who experienced exacerbation of symptoms following cocaine use, but a complete remission of symptoms for hours or days following psilocybin ingestion. One of the patients had moved to a daily treatment regimen, which led to a tolerance to psychedelic effects, but continued relief from symptoms. Wilcox (2014) reported a patient with OCD taking psilocybin every three weeks for the continued abatement of anxiety, intrusive thoughts, and rituals. Hanes (1996) reported a male patient with body dysmorphic disorder (understood as a related disorder in the DSM-5), who typically spent up to $4 \mathrm{~h}$ a day checking his appearance in the mirror, a behavior which caused him significant distress. On multiple occasions after taking Psilocybe mushrooms, his distress was significantly eased, and he no longer saw himself as deformed when looking in the mirror.

An open-label study of psilocybin for OCD (Moreno, Wiegand, Taitano, \& Delgado, 2006) enrolled nine patients with moderate-to-severe symptoms (Storch et al., 2015), who had an average of 3.4 previous treatment failures, and of whom five were unable to work because of their condition. Patients received three escalating doses of psilocybin $(7 \mathrm{mg} /$ $70 \mathrm{~kg}, 14 \mathrm{mg} / 70 \mathrm{~kg}$, and $21 \mathrm{mg} / 70 \mathrm{~kg}$ ), each separated by at least a week, with a very low dose $(1.75 \mathrm{mg} / 70 \mathrm{~kg})$ inserted randomly in a double-blind manner at any point after the first dose. Across the 24-h post-administration monitoring period, all patients experienced marked relief from symptoms, i.e., a $23-100 \%$ reduction from baseline on the YaleBrown Obsessive-Compulsive Scale (YBOCS), in one or more psilocybin session. Two-thirds of patients maintained a $\geq 50 \%$ decrease in YBOCS score at 24 hours for at least one session. Two patients reported symptomatic relief lasting nearly a week, with one patient still in remission at the 6month follow-up. Notably, neither the psilocybin dose, nor the intensity of its subjective effects, predicted the magnitude of change to YBOCS scores.
A number of shortcomings in this study could be addressed in future investigations. Crucially, and in contrast to the bulk of recent clinical research into psilocybin, patients were not provided formal adjunct therapy in advance of and after treatment. There is some evidence to suggest that longer therapist-patient contact before drug sessions increases the probability of a peak experience (Richards, Rhead, DiLeo, Yensen, \& Kurland, 1977). Patient reports and observer recordings have pointed to the beneficial effect of preparatory measures, including the setting of formal intentions, the development of solid rapport with therapists across multiple preparatory sessions, and the placement of drug sessions within a wider therapeutic context (Haijen et al., 2018; Noorani, Garcia-Romeu, Swift, Griffiths, \& Johnson 2018). It has long been recognized that, during the 'psychedelic afterglow', the days and weeks following a psychedelic experience, 'there is a unique opportunity for effective psychotherapeutic work' (Pahnke, Kurland, Unger, Savage, \& Grof, 1970), possibly mediated by an increase in psychological flexibility (Close, Haijen, Watts, Roseman, \& Carhart-Harris, 2020; Davis, Barrett, \& Griffiths, 2020). Although five of the patients of the Moreno study 'readily described their experiences as very psychologically and spiritually enriching', and four reported 'profound positive transcendental experiences', there was no formal measure of mystical experience taken. As well as producing a nonnegligible hallucinogenic effect, the very low dose of psilocybin, which had been intended as a placebo comparator, provided substantial relief from symptoms in four patients. While the dose-independent reduction in symptoms might be partially explained as a result of an expectancy-driven placebo effect, the magnitude and longevity of relief in some patients, as well as low reported magnitudes of placebo effects in OCD (Sugarman, Kirsh, \& Hupper, 2017), suggest some pharmacologically mediated action.

\section{Possible psychedelic mechanisms of action in OCD}

Neurobiologically, both the acute action of psychedelics, and their longer-lasting effects, provide possible accounts of their therapeutic action in OCD, which need not be considered as mutually exclusive.

The cortical $5-\mathrm{HT}_{2 \mathrm{~A}}$ hyper-activation that occurs during the acute psychedelic state is known to lead to alterations in the functioning of the CSTC circuit (Fantegrossi et al., 2008; Nichols, 2004), disrupting the thalamic gating of sensory and cognitive information - resulting not only in perceptual distortions (De Gregorio, Enns, Nunez, Posa, \& Gobbi, 2018; Vollenweider \& Geyer, 2001), but likely also the manifestation of otherwise unconscious psychic material, understood to be a central component of psychedelic, as well as traditional psychoanalytic, psychotherapy (Freud \& Crick, 1999; Grof, 1980). A leading explanation of enduring antidepressive effect of psilocybin is that it functions like a 'reset button' - whereby acute disruption of a maladaptively functioning DMN is followed by post-acute reintegration with a resumption of normal functioning (Carhart-Harris et al., 2017). Functional abnormalities to CSTC circuitry in 
OCD are known to normalize after successful treatment by pharmacotherapy or psychotherapy (Saxena \& Rauch, 2000; Schwartz, Stoessel, Baxter, Martin, \& Phelps, 1996; Van der Straten, Denys, \& van Wingen, 2017), so the psychedelic 'reset' may also be at work in the treatment of OCD, with normal functioning of the CSTC circuitry restored after an acute disruption.

An alternative neurobiological account of obsessional activity in OCD emphasizes the role of dysfunctional activity in the DMN (Beucke et al., 2014; Gonçalves et al., 2017; Koch et al., 2018), a network which is associated with selfreferential cognitive processing (Buckner, Andrews-Hanna, $\&$ Schacter, 2008). Excessive self-referential cognitive activity has long been understood to be present in OCD (Purdon \& Clark, 1999; Salkovskis, 1985). The disruption and reintegration 'reset' in DMN activity that is seen with psilocybin may, in OCD patients, allow the easing of an overly strong, top-down filtering bias, thereby re-establishing normal responsiveness towards the environment. A particularly promising model of the subjective and clinical effects of psychedelics as relaxation of top-down biases, framed in the formal terms of the free energy principle and hierarchical predictive coding, was recently developed by Carhart-Harris \& Friston(2019).

Reductions in compulsivity following successful treatment for OCD may be cast, in part, as restorations to cognitive flexibility - the capacity to stop or switch behaviors to optimize outcomes. OCD populations record various reaction time and accuracy deficits in reversal learning tasks, which track abnormal activity within the OFC, and its connectivity to the wider CSTC loop (Chamberlain et al., 2008; Freyer et al., 2011; Remijnse et al., 2009; Tezcan, Tumkaya, \& Bora, 2017). Both psilocybin and the structurally similar LSD have been demonstrated to enhance reversal learning in rat and primate models (King, Martin, \& Melville, 1974; Roberts \& Bradley, 1967), an effect which antagonist studies with SSRIs suggest may be mediated by their $5-\mathrm{HT}_{2 \mathrm{~A}}$ agonism in the OFC (Boulougouris, Glennon, \& Robbins, 2008; Furr, Lapiz-Bluhm, \& Morilak, 2012).

Despite some support for the role of $5-\mathrm{HT}_{2 \mathrm{~A}}$ receptor binding in the attenuation of these deficiencies related to OCD, the evidence suggests this is a compensatory effect, rather than a normalization of healthy function. Neuroimaging studies of $5-\mathrm{HT}_{2 \mathrm{~A}}$ receptor binding in OCD provide mixed reports, with the largest and most homogenous samples (Simpson et al., 2011) failing to replicate early indications of increased binding in OCD patients relative to controls (Adams et al., 2005), or increased binding within patients correlating to symptom severity (Perani et al., 2008).

The post-psilocybin improvements to OCD symptoms reported in case studies and by Moreno et al. (2006) may also be supported by changes in affective processing. Through 5- $\mathrm{HT}_{2 \mathrm{~A}}$ agonism, psilocybin increases goaldirected behavior towards positive compared to negative cues (Kometer et al., 2012), and reductions to measures of amygdala activity and connectivity in response to negative emotional facial expressions reported in both healthy and clinical populations (Barrett, Doss, Sepeda, Pekar, \&
Griffiths, 2020; Kraehenmann et al., 2015; Kraehenmann et al., 2016; Mertens et al., 2020). Although it remains to be seen whether a similar effect is seen in response to OCD triggers, blunting of emotional reactivity through reduced amygdalar response may also support attenuation of symptoms, interrupting the obsession-compulsion cycle by decreasing its affective/motivational component.

\section{Future research directions}

Research to determine the precise mechanisms and effectiveness of psilocybin in OCD remains stymied by the legal status of psilocybin. Despite its striking clinical effect in a number of indications, as a naturally occurring compound, psilocybin presents a challenging intellectual property space which may temper the enthusiasm of industry for investment of the significant resources required to bring a treatment to market. The stigma associated with psilocybin's restrictive legal classification deters both grant funders and ethics committees from supporting research (Rucker, 2015), while these restrictions hinder the pace of scientific investigation by introducing significant bureaucratic and financial hurdles, meaning only the best-resourced and most determined scientists are able to pursue research. Many of these hurdles are in place whether a lab requires $8 \mathrm{mg}$ of psilocybin for an animal study or $800 \mathrm{mg}$ for a clinical trial. Understanding of the potential role of psilocybin for OCD would be greatly enriched by extending Matsushima et al.'s (2009) mouse study to other rodent models with higher predictive validity (Thompson \& Dulawa, 2017) or which capture other features of OCD (Pittenger et al., 2017). But with entry costs to all psychedelic research so high, it is perhaps not surprising that the psychedelic renaissance has seen animal research using psilocybin trail behind higher-impact and more headlinegrabbing clinical research (Murnane, 2018).

While the efficacy of psilocybin in treating OCD has not been directly compared to that of SSRIs, wider contextual factors favor its further investigation. Up to $90 \%$ of OCD patients have comorbid conditions, including mood, anxiety, and substance use disorders (Ruscio et al., 2010), while comorbid major depressive disorder is associated with decreased likelihood of recovery from OCD (Marcks, Weisberg, Dyck, \& Keller, 2011). The growing evidence in support of psilocybin for treating these conditions suggests that future clinical studies ought to consider permitting the participation of patients with comorbid disorders.

The Arizona lab which completed the original clinical study is now running an expanded, placebo-controlled trial with psilocybin (ClinicalTrials.gov ID: NCT03300947). The inclusion of functional neuroimaging at multiple time points will provide invaluable data about how changes in functional connectivity map onto therapeutic outcomes. Nonetheless, beyond an integrative debriefing after each of the eight sessions, these are not embedded within a wider psychotherapeutic program (Moreno, personal communication). As such, the trial is effectively a test of a novel pharmacotherapy, rather than a combined pharmaco-psychotherapy approach of the style that has generated impressive effect sizes for other 
indications. Considering the evidence outlined above on the afterglow of increased psychological flexibility (and, perhaps, receptivity to therapy) in the post-acute phase, a properly informed understanding of the potential of psilocybin to treat OCD will not be secured before trials are undertaken with an appropriate adjunct psychotherapy.

There are some reasons for optimism about the feasibility of expanding this research. To its credit, the UK government has furthered psilocybin research through Medical Research Council and National Institute of Health Research grants, exploring its potential role in treating depression. Nonetheless, a far more effective course of action would be to remove the excessive restrictions surrounding legitimate uses of psilocybin. Cannabis was moved from Schedule 1 to Schedule 2 of the Misuse of Drugs Regulations within four months in 2018. Doing the same with psilocybin will vastly reduce the financial and bureaucratic hindrances associated with research, while maintaining a level of oversight and control deemed acceptable for drugs with significantly greater harm profiles, including both medical cocaine and diamorphine hydrochloride (heroin).

\section{CONCLUSION}

Given the relative lack of efficacy of current treatment options, OCD and its management remain a global healthcare problem. The current mainstay drug class, SSRIs, come with significant side-effects, with higher doses increasing efficacy only at the cost of a higher side-effect burden (Bloch, McGuire, Landeros-Weisenberger, Leckman, \& Pittenger, 2010). In comparison, psilocybin has been demonstrated to be both physiologically and psychologically safe when taken under the supervision of a clinician, and has very low dependence potential. Given that intractable OCD is an indication for neurosurgery, there is a particularly strong case for the exploration of novel pharmacotherapies, and safe alternative drugs - even those with sensationalized cultural histories - ought to be objectively explored.

Some preclinical evidence, along with a number of case studies and a single controlled trial point towards a possible treatment effect from psilocybin. Moreover, abnormalities at both the receptor and network levels that are implicated in OCD symptomatology are known to be disrupted by psychedelics, marking out potential mechanisms of action. The preliminary safety and efficacy data provide sufficient scientific grounds to justify the expansion of systematic investigation of psilocybin as a treatment of OCD. But while excessive restrictions and political inertia impede such an expansion, dissuading both academia and industry from research, the cost is ultimately borne by patients.

Disclosure statement: The author has previously served in consultancy roles for The Beckley Foundation, Beckley Canopy Therapeutics, and Psilonautica. None were connected in any way to the conception, research, authorship, or publication of this article.
Acknowledgments: The author thanks Valerie Bonnelle, James Rucker, Sam Gandy, Camilla Day and Joe Kinsella for feedback on an earlier draft of the review. Particular thanks are also extended to an anonymous reviewer for a number of invaluable comments.

\section{REFERENCES}

Adams, K. H., Hansen, E. S., Pinborg, L. H., Hasselbalch, S. G., Svarer, C., Holm, S., et al. (2005). Patients with obsessivecompulsive disorder have increased 5-HT2A receptor binding in the caudate nuclei. International Journal of Neuropsychopharmacology, 8(3), 391-401.

Aghajanian, G. K., \& Marek, G. J. (1999). Serotonin, via 5-HT2A receptors, increases EPSCs in layer $\mathrm{V}$ pyramidal cells of prefrontal cortex by an asynchronous mode of glutamate release. Brain Research, 825(1-2), 161-171.

Asbahr, F. R., Garvey, M. A., Snider, L. A., Zanetta, D. M., Elkis, H., \& Swedo, S. E. (2005). Obsessive-compulsive symptoms among patients with Sydenham chorea. Biological Psychiatry, 57(9), 1073-1076.

Barrett, F. S., Doss, M. K., Sepeda, N. D., Pekar, J. J., \& Griffiths, R. R. (2020). Emotions and brain function are altered up to one month after a single high dose of psilocybin. Scientific Reports, 10(1), 1-14.

Barrett, F. S., Johnson, M. W., \& Griffiths, R. R. (2015). Validation of the revised Mystical Experience Questionnaire in experimental sessions with psilocybin. Journal of Psychopharmacology, 29(11), 1182-1190.

Baumeister, D., Barnes, G., Giaroli, G., \& Tracy, D. (2014). Classical hallucinogens as antidepressants? A review of pharmacodynamics and putative clinical roles. Therapeutic Advances in Psychopharmacology, 4(4), 156-169.

Béique, J. C., Imad, M., Mladenovic, L., Gingrich, J. A., \& Andrade, R. (2007). Mechanism of the 5-hydroxytryptamine 2A receptormediated facilitation of synaptic activity in prefrontal cortex. Proceedings of the National Academy of Sciences, 104(23), 9870-9875.

Berney, A., Sookman, D., Leyton, M., Young, S. N., \& Benkelfat, C. (2006). Lack of effects on core obsessive-compulsive symptoms of tryptophan depletion during symptom provocation in remitted obsessive-compulsive disorder patients. Biological Psychiatry, 59(9), 853-857.

Beucke, J. C., Sepulcre, J., Eldaief, M. C., Sebold, M., Kathmann, N., \& Kaufmann, C. (2014). Default mode network subsystem alterations in obsessive-compulsive disorder. The British Journal of Psychiatry, 205(5), 376-382.

Bloch, M. H., McGuire, J., Landeros-Weisenberger, A., Leckman, J. F., \& Pittenger, C. (2010). Meta-analysis of the dose-response relationship of SSRI in obsessive-compulsive disorder. Molecular Psychiatry, 15(8), 850.

Bogenschutz, M. P., Forcehimes, A. A., Pommy, J. A., Wilcox, C. E., Barbosa, P. C. R., \& Strassman, R. J. (2015). Psilocybin-assisted treatment for alcohol dependence: A proof-of-concept study. Journal of Psychopharmacology, 29(3), 289-299.

Boulougouris, V., Glennon, J. C., \& Robbins, T. W. (2008). Dissociable effects of selective 5-HT 2A and 5-HT 2C receptor 
antagonists on serial spatial reversal learning in rats. Neuropsychopharmacology, 33(8), 2007-2019.

Buckner, R. L., Andrews-Hanna, J. R., \& Schacter, D. L. (2008). The brain's default network: anatomy, function, and relevance to disease. Annals of the New York Academy of Sciences, 1124(1).

Carbonaro, T. M., Bradstreet, M. P., Barrett, F. S., MacLean, K. A., Jesse, R., Johnson, M. W., et al. (2016). Survey study of challenging experiences after ingesting psilocybin mushrooms: Acute and enduring positive and negative consequences. Journal of Psychopharmacology, 30(12), 1268-1278.

Carhart-Harris, R., \& Friston, K. (2019). REBUS and the anarchic brain: toward a unified model of the brain action of psychedelics. Pharmacological Reviews, 71(3), 316-344.

Carhart-Harris, R. L. (2019). How do psychedelics work?. Current Opinion in Psychiatry, 32(1), 16-21.

Carhart-Harris, R. L., Bolstridge, M., Day, C. M. J., Rucker, J., Watts, R., Erritzoe, D. E., et al. (2018a). Psilocybin with psychological support for treatment-resistant depression: Sixmonth follow-up. Psychopharmacology, 235(2), 399-408.

Carhart-Harris, R. L., Erritzoe, D., Williams, T., Stone, J. M., Reed, L. J., Colasanti, A., et al. (2012). Neural correlates of the psychedelic state as determined by fMRI studies with psilocybin. Proceedings of the National Academy of Sciences, 109(6), 2138-2143.

Carhart-Harris, R. L., Muthukumaraswamy, S., Roseman, L., Kaelen, M., Droog, W., Murphy, K., et al. (2016). Neural correlates of the LSD experience revealed by multimodal neuroimaging. Proceedings of the National Academy of Sciences, 113(17), 4853-4858.

Carhart-Harris, R. L., Roseman, L., Bolstridge, M., Demetriou, L., Pannekoek, J. N., Wall, M. B., et al. (2017). Psilocybin for treatment-resistant depression: fMRI-measured brain mechanisms. Scientific Reports, 7(1), 13187.

Carhart-Harris, R. L., Roseman, L., Haijen, E., Erritzoe, D., Watts, R., \& Branchi, I., et al. (2018b). Psychedelics and the essential importance of context. Journal of Psychopharmacology, 32(7), 725-731.

Carmin, C. N., Wiegartz, P. S., Yunus, U., \& Gillock, K. L. (2002). Treatment of late-onset OCD following basal ganglia infarct. Depression and Anxiety, 15(2), 87-90.

Chamberlain, S. R., Menzies, L., Hampshire, A., Suckling, J., Fineberg, N. A., del Campo, N., et al. (2008). Orbitofrontal dysfunction in patients with obsessive-compulsive disorder and their unaffected relatives. Science, 321(5887), 421-422.

Close, J. B., Haijen, E. C., Watts, R., Roseman, L., \& Carhart-Harris, R. L. (2020). Psychedelics and psychological flexibility - Results of a prospective web-survey using the Acceptance and Action Questionnaire II. Journal of Contextual Behavioral Science, 16, $37-44$.

Coluccia, A., Fagiolini, A., Ferretti, F., Pozza, A., Costoloni, G., Bolognesi, S., et al. (2016). Adult obsessive-compulsive disorder and quality of life outcomes: A systematic review and metaanalysis. Asian Journal of Psychiatry, 22, 41-52.

Davis, A. K., Barrett, F. S., \& Griffiths, R. R. (2020). Psychological flexibility mediates the relations between acute psychedelic effects and subjective decreases in depression and anxiety. Journal of Contextual Behavioral Science, 15, 39-45.
De Gregorio, D., Enns, J. P., Nunez, N. A., Posa, L., \& Gobbi, G. (2018). Hallucinogens: Mechanism of action and potential therapeutic applications in mood disorders. Psychedelic Neuroscience, 242, 69.

Del Casale, A., Kotzalidis, G. D., Rapinesi, C., Serata, D., Ambrosi, E., Simonetti, A., et al. (2011). Functional neuroimaging in obsessive-compulsive disorder. Neuropsychobiology, 64(2), 61-85.

Denys, D. (2006). Pharmacotherapy of obsessive-compulsive disorder and obsessive-compulsive spectrum disorders. Psychiatric Clinics of North America, 29(2), 553-584.

Denys, D., van der Wee, N., van Megen, H. J., \& Westenberg, H. G. (2003). A double blind comparison of venlafaxine and paroxetine in obsessive-compulsive disorder. Journal of Clinical Psychopharmacology, 23(6), 568-575.

Fantegrossi, W. E., Reissig, C. J., Katz, E. B., Yarosh, H. L., Rice, K. C., \& Winter, J. C. (2008). Hallucinogen-like effects of N, Ndipropyltryptamine (DPT): Possible mediation by serotonin 5HT1A and 5-HT2A receptors in rodents. Pharmacology Biochemistry and Behavior, 88(3), 358-365.

Fisher, P. L., \& Wells, A. (2005). How effective are cognitive and behavioral treatments for obsessive-compulsive disorder? A clinical significance analysis. Behaviour Research and Therapy, 43(12), 1543-1558.

Fontenelle, L. F., Mendlowicz, M. V., \& Versiani, M. (2006). The descriptive epidemiology of obsessive-compulsive disorder. Progress in Neuro-Psychopharmacology and Biological Psychiatry, 30(3), 327-337.

Freud, S., \& Crick, J. (1999). Sigmund freud the interpretation of dreams. Trans. Joyce Crick. Oxford: Oxford University Press.

Freyer, T., Klöppel, S., Tüscher, O., Kordon, A., Zurowski, B., Kuelz, A. K., et al. (2011). Frontostriatal activation in patients with obsessive-compulsive disorder before and after cognitive behavioral therapy. Psychological Medicine, 41(1), 207-216.

Furr, A., Lapiz-Bluhm, M. D., \& Morilak, D. A. (2012). 5-HT2A receptors in the orbitofrontal cortex facilitate reversal learning and contribute to the beneficial cognitive effects of chronic citalopram treatment in rats. International Journal of Neuropsychopharmacology, 15(9), 1295-1305.

Garcia-Romeu, A., Griffiths, R. R., \& Johnson, W. M. (2014). Psilocybin-occasioned mystical experiences in the treatment of tobacco addiction. Current Drug Abuse Reviews, 7(3), 157-164.

Gonçalves, Ó. F., Soares, J. M., Carvalho, S., Leite, J., Ganho-Ávila, A., Fernandes-Gonçalves, A., et al. (2017). Patterns of default mode network deactivation in obsessive compulsive disorder. Scientific Reports, 7, 44468.

Graat, I., Figee, M., \& Denys, D. (2017). Neurotransmitter dysregulation in OCD. In C. Pittenger (Ed.), Obsessive-compulsive disorder: Phenomenology, pathophysiology, and treatment. Oxford University Press.

Grassi, L., Caruso, R., Hammelef, K., Nanni, M. G., \& Riba, M. (2014). Efficacy and safety of pharmacotherapy in cancerrelated psychiatric disorders across the trajectory of cancer care: A review. International Review of Psychiatry, 26(1), 44-62.

Griffiths, R. R., Johnson, M. W., Carducci, M. A., Umbricht, A., Richards, W. A., Richards, B. D., et al. (2016). Psilocybin produces substantial and sustained decreases in depression and anxiety in patients with life-threatening cancer: A randomized 
double-blind trial. Journal of Psychopharmacology, 30(12), 1181-1197.

Griffiths, R. R., Johnson, M. W., Richards, W. A., Richards, B. D., McCann, U., \& Jesse, R. (2011). Psilocybin occasioned mysticaltype experiences: Immediate and persisting dose-related effects. Psychopharmacology, 218(4), 649-665.

Grinspoon, L., \& Bakalar, J. B. (1979). Psychedelic drugs reconsidered (pp. 221-223). New York: Basic Books.

Grob, C. S., Danforth, A. L., Chopra, G. S., Hagerty, M., McKay, C. R., Halberstadt, A. L., et al. (2011). Pilot study of psilocybin treatment for anxiety in patients with advanced-stage cancer. Archives of General Psychiatry, 68(1), 71-78.

Grof, S., \& Psychotherapy, L. S. D. (1980). Pomona. CA: Hunter House.

Haijen, E. C., Kaelen, M., Roseman, L., Timmermann, C., Kettner, H., Russ, S., et al. (2018). Predicting responses to psychedelics: A prospective study. Frontiers in Pharmacology, 9, 897.

Halberstadt, A. L., \& Geyer, M. A. (2011). Multiple receptors contribute to the behavioral effects of indoleamine hallucinogens. Neuropharmacology, 61(3), 364-381.

Halberstadt, A. L., Koedood, L., Powell, S. B., \& Geyer, M. A. (2011). Differential contributions of serotonin receptors to the behavioral effects of indoleamine hallucinogens in mice. Journal of Psychopharmacology, 25(11), 1548-1561.

Hanes, K. R. (1996). Serotonin, psilocybin, and body dysmorphic disorder: A case report. Journal of Clinical Psychopharmacology, 16(2), 188-189.

Hartogsohn, I. (2016). Set and setting, psychedelics and the placebo response: An extra-pharmacological perspective on psychopharmacology. Journal of Psychopharmacology, 30(12), 12591267.

Hasler, F., Bourquin, D., Brenneisen, R., Bär, T., \& Vollenweider, F. X. (1997). Determination of psilocin and 4-hydroxyindole-3acetic acid in plasma by HPLC-ECD and pharmacokinetic profiles of oral and intravenous psilocybin in man. Pharmaceutica Acta Helvetiae, 72(3), 175-184.

Hasler, F., Grimberg, U., Benz, M. A., Huber, T., \& Vollenweider, F. X. (2004). Acute psychological and physiological effects of psilocybin in healthy humans: A double-blind, placebocontrolled dose-effect study. Psychopharmacology, 172(2), 145-156.

Hendricks, P. S., Thorne, C. B., Clark, C. B., Coombs, D. W., \& Johnson, M. W. (2015). Classic psychedelic use is associated with reduced psychological distress and suicidality in the United States adult population. Journal of Psychopharmacology, 29(3), 280-288.

Hofmann, A., Frey, A., Ott, H., Petrzilka, T., \& Troxler, F. (1958). Konstitutionsaufklärung und synthese von psilocybin. Experientia, 14(11), 397-399.

Hofmann, A., \& Schultes, R. E. (1979). Plants of the gods. Van der Mark Editions.

Horita, A. (1963). Some biochemical studies on psilocybin and psilogin. Journal of Neuropsychiatry, 4, 270-273.

Johansen, P. Ø., \& Krebs, T. S. (2015). Psychedelics not linked to mental health problems or suicidal behavior: A population study. Journal of Psychopharmacology, 29(3), 270-279.
Johnson, M. W., Garcia-Romeu, A., \& Griffiths, R. R. (2017). Longterm follow-up of psilocybin-facilitated smoking cessation. The American Journal of Drug and Alcohol Abuse, 43(1), 55-60.

Johnson, M. W., Richards, W. A., \& Griffiths, R. R. (2008). Human hallucinogen research: Guidelines for safety. Journal of Psychopharmacology, 22(6), 603-620.

King, A. R., Martin, I. L., \& Melville, K. A. (1974). Reversal learning enhanced by lysergic acid diethylamide (LSD): Concomitant rise in brain 5-hydroxytryptamine levels. British Journal of Pharmacology, 52(3), 419.

Koch, K., Reeß, T. J., Rus, O. G., Gürsel, D. A., Wagner, G., Berberich, G., et al. (2018). Increased default mode network connectivity in obsessive-compulsive disorder during reward processing. Frontiers in Psychiatry, 9, 254.

Kometer, M., Schmidt, A., Bachmann, R., Studerus, E., Seifritz, E., \& Vollenweider, F. X. (2012). Psilocybin biases facial recognition, goal-directed behavior, and mood state toward positive relative to negative emotions through different serotonergic subreceptors. Biological Psychiatry, 72(11), 898-906.

Kraehenmann, R., Preller, K. H., Scheidegger, M., Pokorny, T., Bosch, O. G., Seifritz, E., et al. (2015). Psilocybin-induced decrease in amygdala reactivity correlates with enhanced positive mood in healthy volunteers. Biological Psychiatry, 78(8), 572-581.

Kraehenmann, R., Schmidt, A., Friston, K., Preller, K. H., Seifritz, E., \& Vollenweider, F. X. (2016). The mixed serotonin receptor agonist psilocybin reduces threat-induced modulation of amygdala connectivity. NeuroImage: Clinical, 11, 53-60.

Krebs, T. S., \& Johansen, P. Ø. (2013). Psychedelics and mental health: A population study. PLoS One, 8(8), e63972.

Leary, T., Litwin, G. H., \& Metzner, R. (1963). Reactions to psilocybin administered in a supportive environment. Journal of Nervous and Mental Disease, 137, 561-573.

Lee, M. A., \& Shlain, B. (1985). Acid dreams: The CIA, LSD, and the sixties rebellion. Grove Press.

Leonard, H. L., \& Rapoport, J. L. (1987). Relief of obsessivecompulsive symptoms by LSD and psilocin. American Journal of Psychiatry, 144(9), 1239-1240.

Ly, C., Greb, A. C., Cameron, L. P., Wong, J. M., Barragan, E. V., Wilson, P. C., et al. (2018). Psychedelics promote structural and functional neural plasticity. Cell Reports, 23(11), 3170-3182.

Madsen, M. K., Fisher, P. M., Burmester, D., Dyssegaard, A., Stenbæk, D. S., Kristiansen, S., et al. (2019). Psychedelic effects of psilocybin correlate with serotonin $2 \mathrm{~A}$ receptor occupancy and plasma psilocin levels. Neuropsychopharmacology, 44(7), 1328.

Majić, T., Schmidt, T. T., \& Gallinat, J. (2015). Peak experiences and the afterglow phenomenon: When and how do therapeutic effects of hallucinogens depend on psychedelic experiences?. Journal of Psychopharmacology, 29(3), 241-253.

Marcks, B. A., Weisberg, R. B., Dyck, I., \& Keller, M. B. (2011). Longitudinal course of obsessive-compulsive disorder in patients with anxiety disorders: A 15-year prospective follow-up study. Comprehensive Psychiatry, 52(6), 670-677.

Matsushima, Y., Shirota, O., Kikura-Hanajiri, R., Goda, Y., \& Eguchi, F. (2009). Effects of Psilocybe argentipes on marbleburying behavior in mice. Bioscience, Biotechnology, and Biochemistry, 73(8), 1866-1868. 
McDougle, C. J., Epperson, C. N., Pelton, G. H., Wasylink, S., \& Price, L. H. (2000). A double-blind, placebo-controlled study of risperidone addition in serotonin reuptake inhibitor-refractory obsessive-compulsive disorder. Archives of General Psychiatry, 57(8), 794-801.

Menzies, L., Chamberlain, S. R., Laird, A. R., Thelen, S. M., Sahakian, B. J., \& Bullmore, E. T. (2008). Integrating evidence from neuroimaging and neuropsychological studies of obsessive-compulsive disorder: The orbitofronto-striatal model revisited. Neuroscience \& Biobehavioral Reviews, 32(3), 525-549.

Mertens, L. J., Wall, M. B., Roseman, L., Demetriou, L., Nutt, D. J., \& Carhart-Harris, R. L. (2020). Therapeutic mechanisms of psilocybin: Changes in amygdala and prefrontal functional connectivity during emotional processing after psilocybin for treatment-resistant depression. Journal of Psychopharmacology, 34(2), 167-180.

Milad, M. R., \& Rauch, S. L. (2012). Obsessive-compulsive disorder: Beyond segregated cortico-striatal pathways. Trends in Cognitive Sciences, 16(1), 43-51.

Moreno, F. A., \& Delgado, P. L. (1997). Hallucinogen-induced relief of obsessions and compulsions. American Journal of Psychiatry, 154(7), 1037-1038.

Moreno, F. A., Wiegand, C. B., Taitano, E. K., \& Delgado, P. L. (2006). Safety, tolerability, and efficacy of psilocybin in 9 patients with obsessive-compulsive disorder. Journal of Clinical Psychiatry, 67(11), 1735-1740.

Müller, F., Dolder, P. C., Schmidt, A., Liechti, M. E., \& Borgwardt, S. (2018). Altered network hub connectivity after acute LSD administration. NeuroImage: Clinical, 18, 694-701.

Murnane, K. S. (2018). The renaissance in psychedelic research: What do preclinical models have to offer. In Progress in brain research (Vol. 242, pp. 25-67). Elsevier.

Muschamp, J. W., Regina, M. J., Hull, E. M., Winter, J. C., \& Rabin, R. A. (2004). Lysergic acid diethylamide and [-]-2, 5-dimethoxy-4-methylamphetamine increase extracellular glutamate in rat prefrontal cortex. Brain Research, 1023(1), 134-140.

Muthukumaraswamy, S. D., Carhart-Harris, R. L., Moran, R. J., Brookes, M. J., Williams, T. M., Errtizoe, D., et al. (2013). Broadband cortical desynchronization underlies the human psychedelic state. Journal of Neuroscience, 33(38), 1517115183.

Nakao, T., Okada, K., \& Kanba, S. (2014). Neurobiological model of obsessive-compulsive disorder: Evidence from recent neuropsychological and neuroimaging findings. Psychiatry and Clinical Neurosciences, 68(8), 587-605.

Nichols, D. E. (2004). Hallucinogens. Pharmacology \& Therapeutics, 101(2), 131-181.

Nichols, D. E. (2016). Psychedelics. Pharmacological reviews, 68(2), 264-355.

Noorani, T., Garcia-Romeu, A., Swift, T. C., Griffiths, R. R., \& Johnson, M. W. (2018). Psychedelic therapy for smoking cessation: Qualitative analysis of participant accounts. Journal of Psychopharmacology, 32(7), 756-769.

Ostuzzi, G., Benda, L., Costa, E., \& Barbui, C. (2015). Efficacy and acceptability of antidepressants on the continuum of depressive experiences in patients with cancer: Systematic review and meta-analysis. Cancer Treatment Reviews, 41(8), 714-724.
Pahnke, W. N. (1966). Drugs and mysticism. International Journal of Parapsychology, 8(2), 295-313.

Pahnke, W. N. (1969). Psychedelic drugs and mystical experience. International Psychiatry Clinics, 5(4), 149.

Pahnke, W. N., Kurland, A. A., Unger, S., Savage, C., \& Grof, S. (1970). The experimental use of psychedelic (LSD) psychotherapy. Jama, 212(11), 1856-1863.

Palhano-Fontes, F., Andrade, K. C., Tofoli, L. F., Santos, A. C., Crippa, J. A. S., Hallak, J. E., et al. (2015). The psychedelic state induced by ayahuasca modulates the activity and connectivity of the default mode network. PloS One, 10(2), e0118143.

Parent, M., Wallman, M. J., Gagnon, D., \& Parent, A. (2011). Serotonin innervation of basal ganglia in monkeys and humans. Journal of Chemical Neuroanatomy, 41(4), 256-265.

Perani, D., Garibotto, V., Gorini, A., Moresco, R. M., Henin, M., Panzacchi, A., et al. (2008). In vivo PET study of 5HT2A serotonin and $\mathrm{D} 2$ dopamine dysfunction in drug-naive obsessivecompulsive disorder. NeuroImage, 42(1), 306-314.

Pittenger, C., \& Bloch, M. H. (2014). Pharmacological treatment of obsessive-compulsive disorder. Psychiatria Clinica, 37(3), 375-391.

Pittenger, C., Dulawa, S., \& Thompson, S. (2017). Animal models in OCD: A conceptual framework. In C. Pittenger (Ed.), Obsessivecompulsive disorder: Phenomenology, pathophysiology, and treatment. Oxford University Press.

Purdon, C., \& Clark, D. A. (1999). Metacognition and obsessions. Clinical Psychology \& Psychotherapy: An International Journal of Theory \& Practice, 6(2), 102-110.

Quednow, B. B., Kometer, M., Geyer, M. A., \& Vollenweider, F. X. (2012). Psilocybin-induced deficits in automatic and controlled inhibition are attenuated by ketanserin in healthy human volunteers. Neuropsychopharmacology, 37(3), 630.

Ray, T. S. (2010). Psychedelics and the human receptorome. PLoS One, 5(2), e9019.

Remijnse, P. L., Nielen, M. M. A., Van Balkom, A. J. L. M., Hendriks, G. J., Hoogendijk, W. J., Uylings, H. B. M., et al. (2009). Differential frontal-striatal and paralimbic activity during reversal learning in major depressive disorder and obsessive-compulsive disorder. Psychological Medicine, 39(9), 1503-1518.

Richards, W. A., Rhead, J. C., DiLeo, F. B., Yensen, R., \& Kurland, A. A. (1977). The peak experience variable in DPT-assisted psychotherapy with cancer patients. Journal of Psychedelic Drugs, 9(1), 1-10.

Roberts, M. H. T., \& Bradley, P. B. (1967). Studies on the effects of drugs on performance of a delayed discrimination. Physiology \& Behavior, 2(4), 389-397.

Roseman, L., Nutt, D. J., \& Carhart-Harris, R. L. (2018). Quality of acute psychedelic experience predicts therapeutic efficacy of psilocybin for treatment-resistant depression. Frontiers in Pharmacology, 8, 974.

Ross, S., Bossis, A., Guss, J., Agin-Liebes, G., Malone, T., Cohen, B., et al. (2016). Rapid and sustained symptom reduction following psilocybin treatment for anxiety and depression in patients with life-threatening cancer: A randomized controlled trial. Journal of Psychopharmacology, 30(12), 1165-1180.

Rucker, J. J. (2015). Psychedelic drugs should be legally reclassified so that researchers can investigate their therapeutic potential. BMJ, 350, h2902. 
Rucker, J. J., Jelen, L. A., Flynn, S., Frowde, K. D., \& Young, A. H. (2016). Psychedelics in the treatment of unipolar mood disorders: A systematic review. Journal of Psychopharmacology, 30(12), 1220-1229.

Ruscio, A. M., Stein, D. J., Chiu, W. T., \& Kessler, R. C. (2010). The epidemiology of obsessive-compulsive disorder in the national comorbidity survey replication. Molecular Psychiatry, 15(1), 53.

Sakai, Y., Narumoto, J., Nishida, S., Nakamae, T., Yamada, K., Nishimura, T., et al. (2011). Corticostriatal functional connectivity in non-medicated patients with obsessive-compulsive disorder. European Psychiatry, 26(7), 463-469.

Salkovskis, P. M. (1985). Obsessional-compulsive problems: A cognitive-behavioural analysis. Behaviour Research and Therapy, 23(5), 571-583.

Saxena, S., \& Rauch, S. L. (2000). Functional neuroimaging and the neuroanatomy of obsessive-compulsive disorder. Psychiatric Clinics of North America, 23(3), 563-586.

Schwartz, J. M., Stoessel, P. W., Baxter, L. R., Martin, K. M., \& Phelps, M. E. (1996). Systematic changes in cerebral glucose metabolic rate after successful behavior modification treatment of obsessive-compulsive disorder. Archives of General Psychiatry, 53(2), 109-113.

Simpson, H. B., Slifstein, M., Bender , Jr, J., Xu, X., Hackett, E., Maher, M. J., et al. (2011). Serotonin 2A receptors in obsessive-compulsive disorder: A positron emission tomography study with [11C] MDL 100907. Biological Psychiatry, 70(9), 897-904.

Storch, E. A., De Nadai, A. S., do Rosário, M. C., Shavitt, R. G., Torres, A. R., Ferrão, Y. A., et al. (2015). Defining clinical severity in adults with obsessive-compulsive disorder. Comprehensive Psychiatry, 63, 30-35.

Studerus, E., Kometer, M., Hasler, F., \& Vollenweider, F. X. (2011). Acute, subacute and long-term subjective effects of psilocybin in healthy humans: A pooled analysis of experimental studies. Journal of Psychopharmacology, 25(11), 14341452.

Sugarman, M. A., Kirsch, I., \& Huppert, J. D. (2017). Obsessivecompulsive disorder has a reduced placebo (and antidepressant) response compared to other anxiety disorders: A metaanalysis. Journal of Affective Disorders, 218, 217-226.

Tagliazucchi, E., Roseman, L., Kaelen, M., Orban, C., Muthukumaraswamy, S. D., Murphy, K., et al. (2016). Increased global functional connectivity correlates with LSD-induced ego dissolution. Current Biology, 26(8), 1043-1050.

Taylor, G. T., Lerch, S., \& Chourbaji, S. (2017). Marble burying as compulsive behaviors in male and female mice. Acta Neurobiologiae Experimentalis, 77(3), 254-260.
Tezcan, D., Tumkaya, S., \& Bora, E. (2017). Reversal learning in patients with obsessive-compulsive disorder (OCD) and their unaffected relatives: Is orbitofrontal dysfunction an endophenotype of OCD?. Psychiatry Research, 252, 231-233.

Thobois, S., Jouanneau, E., Bouvard, M., \& Sindou, M. (2004). Obsessive-compulsive disorder after unilateral caudate nucleus bleeding. Acta Neurochirurgica, 146(9), 1027-1031.

Thompson, S., \& Dulawa, S. (2017). Pharmacological and behavioral rodent models of OCD. In C. Pittenger (Ed.), Obsessivecompulsive disorder: Phenomenology, pathophysiology, and treatment. Oxford University Press.

Thorsen, A. L., Hagland, P., Radua, J., Mataix-Cols, D., Kvale, G., Hansen, B., et al. (2018). Emotional processing in obsessivecompulsive disorder: A systematic review and meta-analysis of 25 functional neuroimaging studies. Biological Psychiatry: Cognitive Neuroscience and Neuroimaging, 3(6), 563-571.

Van der Straten, A. L., Denys, D., \& van Wingen, G. A. (2017). Impact of treatment on resting cerebral blood flow and metabolism in obsessive compulsive disorder: A meta-analysis. Scientific Reports, 7(1), 17464.

Vollenweider, F. X., \& Geyer, M. A. (2001). A systems model of altered consciousness: Integrating natural and drug-induced psychoses. Brain Research Bulletin, 56(5), 495-507.

Vollenweider, F. X., \& Kometer, M. (2010). The neurobiology of psychedelic drugs: Implications for the treatment of mood disorders. Nature Reviews Neuroscience, 11(9), 642.

Vollenweider, F. X., Vollenweider-Scherpenhuyzen, M. F., Bäbler, A., Vogel, H., \& Hell, D. (1998). Psilocybin induces schizophrenia-like psychosis in humans via a serotonin-2 agonist action. NeuroReport, 9(17), 3897-3902.

Waterhouse, E. G., An, J. J., Orefice, L. L., Baydyuk, M., Liao, G. Y., Zheng, K., et al. (2012). BDNF promotes differentiation and maturation of adult-born neurons through GABAergic transmission. Journal of Neuroscience, 32(41), 14318-14330.

Welter, M. L., Burbaud, P., Fernandez-Vidal, S., Bardinet, E., Coste, J., Piallat, B., et al. (2011). Basal ganglia dysfunction in OCD: Subthalamic neuronal activity correlates with symptoms severity and predicts high-frequency stimulation efficacy. Translational Psychiatry, 1(5), e5.

Westenberg, H. G., Fineberg, N. A., \& Denys, D. (2007). Neurobiology of obsessive-compulsive disorder: Serotonin and beyond. CNS Spectrums, 12(S3), 14-27.

Wilcox, J. A. (2014). Psilocybin and obsessive compulsive disorder. Journal of Psychoactive Drugs, 46(5), 393-395.

Winstock, A. R., Barratt, M. J., Maier, L. J., \& Ferris, J. A. (2018). Global drug survey (GDS) 2018. Key findings report. https:// www.globaldrugsurvey.com/gds-2018/. 